\title{
Bringt die endovaskuläre Therapie Vorteile gegenüber der systemischen Lyse?
}

Fragestellung: Wie gut sind die Rekanalisierungsquoten bei verschiedenen Devices zur intraarteriellen Thrombektomie bei Patienten mit akutem ischämischem Insult?

Hintergrund: Mit Ausnahme der kürzlich publizierten Studien zur Thrombektomie im Vergleich zur systemischen Lyse mit rtPA beim akuten ischämischen Insult, gibt es eine Reihe von $\mathrm{Be}$ obachtungsstudien, bei denen die Rekanalisierungsraten mit unterschiedlichen Devices untersucht wurden. Die Autoren führten eine systematische Literaturrecherche und eine MetaAnalyse der prospektiven Studien durch, in denen die Rekanalisierungsraten und der Outcome bei unterschiedlichen Thrombektomie-Devices beim akuten Schlaganfall untersucht wurden.

Patienten und Methodik: Die Autoren identifizierten 16 Studien. Bei vier wurde das Merci-Device (357 Patienten), bei acht das Penumbra-Device (455 Patienten) und bei vier das SolitaireDevice (113 Patienten) eingesetzt. Die mittlere prozentuale Dauer betrug für das Merci-Device 120 Minuten. Die mittlere Zeit von der Punktion in der Leiste bis zur Rekanalisation war 64,6 Minuten für Penumbra und 54,7 Minuten für Solitaire. Eine erfolgreiche Rekanalisierung

Almekhlafi MA, Menon BK, Freiheit EA et al. A meta-analysis of observational intra-arterial stroke therapy studies using the Merci device, Penumbra system, and retrievable stents. Am J Neuroradiol 2013; 34: 140-5 fand sich bei $59 \%$ der Patienten die mit dem Merci-Device behandelt wurden, bei $86 \%$ mit dem Penumbra-System und $93 \%$ beim SolitaireDevice. Eine funktionelle Unabhängigkeit, definiert als ein Wert auf der modifizier-

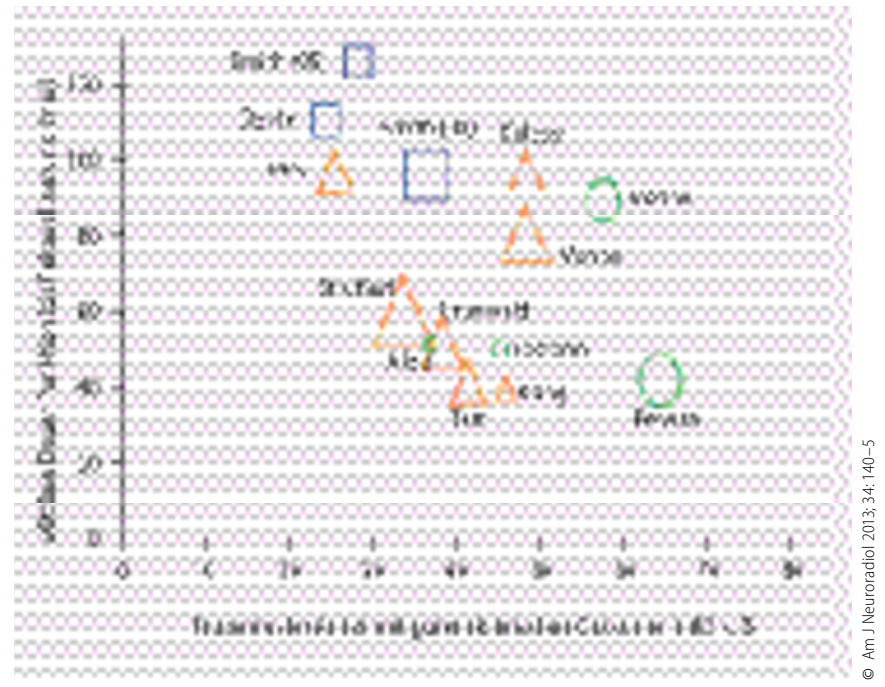

1 Relation zwischen mittlerer Dauer des Eingriffs bis zur Rekanalisierung und funktionellem Outcome.

ten Rankin-Skala von $\leq 2$, erreichten $31,5 \%$ der Patienten mit dem Merci-Device, 36,6\% mit dem Penumbra-Device und $46,9 \%$ mit dem Solitaire-Device ( Abb. 1).

Schlussfolgerungen: Stent-Retriever wie das Solitaire-Device haben höhere Rekanalisierungsraten und einen besseren Outcome im Vergleich zu dem Merci- und Penumbra-System.

\section{- Kommentar von Hans-Christoph Diener, Essen}

\section{Die Effektivität moderner Stent-Retriever weiter prüfen}

Die drei vor kurzem publizierten Studien zur Thrombektomie im Vergleich zur systemischen Thrombolyse waren sehr enttäuschend, da alle drei Studien negativ waren. Allerdings benutzen die drei Studien ganz überwiegend die altmodischeren Merci- und Penumbra-Devices. Das Solitaire-Device hat in der Zwischenzeit ebenso wie das Trevo-Device gezeigt, dass es bezüglich Rekanalisierung und Outcome dem Merci-Device statistisch signifikant überlegen ist. Diese Metaanalyse legt auch nahe, dass das Solitaire-Device wirksamer ist sowohl bezüglich Rekanalisierung wie auch bezüglich des klinischen Outcomes. Daher sollten im Moment nach Möglichkeit alle Patienten die für eine Thrombektomie infrage kommen, in eine der jetzt beginnenden aktuellen Studien zum Einsatz moderner Stent-Retriever im Vergleich zur systemischen Thrombolyse eingeschlossen werden.

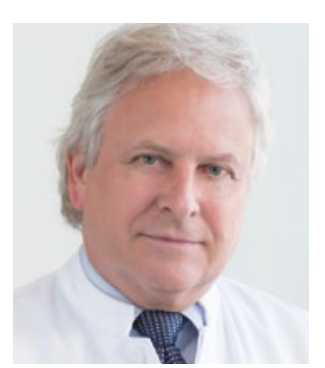

Prof. Dr. med. Hans-Christoph Diener, Essen

Direktor der Klinik für Neurologie, Universitätsklinikum Essen

E-Mail:h.diener@uni-essen.de 